\title{
Estenosis faríngea secundaria a adenoamigdalectomía. ¿Cómo la resolvemos?
}

\author{
Pharyngeal stenosis secondary to adenotonsillectomy. How do we solve it?
}

Carmen Gloria Morovic I', Karen Goldschmied A², Daniela Rojas P³ , Ignacio Cifuentes $\mathbf{0}^{4}$.

\section{RESUMEN}

La estenosis faríngea es una complicación muy poco frecuente de la adenoamigdalectomía. Consiste en un estrechamiento de la vía aerodigestiva superior secundaria a la adhesión parcial o total de las estructuras que componen la orofaringe producto de una disección extensa al realizar amigdalectomía. El objetivo de este trabajo es presentar tres casos clínicos tratamiento y correspondiente técnica quirúrgica. Se describen tres pacientes operados durante la edad preescolar de adenoamigdalectomía, que cursaron en el posoperatorio con estenosis faríngea. Todos presentaron roncopatía severa, respiración oral y resonancia hiponasal. La nasofibroscopía evidenció estrechez faríngea en distintos grados. A todos se les realizó resección del tejido cicatricial y faringoplastía con colgajo miomucoso y posterior rehabilitación con bulbo faríngeo. El uso de colgajos faríngeos posterior a la liberación de adherencias posadenoamigdalectomía permite aportar tejido sano a zonas cruentas, evitando la formación de nuevas adherencias y restituyendo la permeabilidad oronasal. El bulbo faríngeo es fundamental para mantener un apropiado lumen y manejo de la cicatrización en el posoperatorio. La técnica de colgajo faríngeo lateral, que interpone mucosa faríngea sana para la cobertura del lecho cruento, constituye una alternativa quirúrgica eficaz para resolver la estenosis faríngea posquirúrgica.

Palabras clave: Estenosis faríngea, colgajo faríngeo, adenoamigdalectomía, complicaciones.

\begin{abstract}
Pharyngeal stenosis is a very rare complication of adenotonsillectomy. It is caused by a narrowing of the upper aerodigestive pathway secondary to the total or partial adhesion of the retropharyngeal structures as a result of extensive dissection during tonsillectomy. Our aim is to present three consecutive clinical cases of pharyngeal stenosis
\end{abstract}

\footnotetext{
Cirugía Plástica, Hospital Luis Calvo Mackenna, Santiago, Chile.

2 Fonoaudiólogia, Hospital Luis Calvo Mackenna, Santiago Chile.

3 Cirugía Infantil, Hospital Luis Calvo Mackenna, Universidad de Chile, Santiago, Chile.

4 Cirugía General, Pontificia Universidad Católica de Chile, Santiago, Chile.
}

Los autores declaran no tener conflictos de interés.

Recibido el 8 de enero, 2019. Aceptado el 28 de junio, 2019. 
after adenotonsillectomy, discuss its treatment and corresponding surgical technique. We present three infants with adenotonsillectomy with postoperative pharyngeal stenosis. All had severe snoring, oral breathing and hyponasal resonance. The nasofibroscopy showed pharyngeal obliteration in several degrees. All patients underwent scar tissue resection and pharyngoplasty with myomucosal flap and subsequent use of pharyngeal bulb. The use of pharyngeal flaps after release of pharyngeal adhesions allows to provide healthy tissue to row areas, avoiding new postoperative adhesions and providing adequate oronasal permeability. The pharyngeal bulb is essential to maintain permeability and management of scarring in the postoperative period. The technique of lateral pharyngeal flap, which aims to interpose healthy pharyngeal mucosa to cover the bloody bed, is an effective therapeutic alternative to solve post-surgical pharyngeal stenosis.

Key words: Pharyngeal stenosis, pharyngeal flap, adenotonsillectomy, complications.

\section{INTRODUCCIÓN}

La cirugía de adenoides y amígdalas corresponde a uno de los procedimientos quirúrgicos realizados con mayor frecuencia por los otorrinolaringólogos y una de las más comunes en la población pediátrica. Las principales indicaciones son la hiperplasia amigdalina con obstrucción y la amigdalitis crónica ${ }^{1,2}$. A pesar de ser considerado un procedimiento quirúrgico relativamente sencillo, la adenoamigdalectomía no está exenta de complicaciones, siendo la hemorragia y las náuseas las más frecuentes. De manera incidental, se han presentado casos de estenosis faríngea secundaria a este procedimiento quirúrgico con una razón estimada de 3/100.000 cirugías 3 .

La estenosis faríngea se ha definido como el estrechamiento de la comunicación normal entre la nasofaringe y la orofaringe, siendo el resultado de la fusión parcial o total de los pilares amigdalinos, base de lengua y/o paladar blando a la pared posterior de la faringe. Se produce principalmente por adherencia frente a una exposición amplia de tejido cruento. La presentación clínica incluye síntomas como obstrucción nasal, respiración oral, roncopatía, cambios en la voz y/o disfagia de aparición insidiosa dentro de las primeras semanas posteriores a la cirugía. Se han descrito múltiples alternativas de tratamiento, incluyendo la liberación de adhesiones, infiltración de corticoides, y el uso de colgajos faríngeos ${ }^{4-6}$. El objetivo de este estudio es dar a conocer la estenosis faríngea posadenoamigdalectomía, su presentación clínica y tratamiento quirúrgico.

Se reportan 3 casos consecutivos en quienes se realiza adenoamigdalectomía en etapa preescolar, sin complicaciones descritas en sus protocolos. Todos evolucionan con estenosis orofaríngea, que se resuelven quirúrgicamente mediante liberación de adherencias y colgajos faríngeos. Los síntomas que originaron la derivación a nuestro centro fueron roncopatía severa, disfagia lógica, respiración oral y resonancia hiponasal.

\section{CASO CLÍNICO 1}

Paciente de sexo masculino en quien se realiza adenoamigdalectomía a los 3 años de edad. Evoluciona a las dos semanas con roncopatía severay disfagia lógica, secundaria a la fusión parcial del lecho cruento posquirúrgico. Inicialmente se realiza una liberación de sinequias por su otorrinolaringólogo. Dada la persistencia de los síntomas se deriva a nuestro centro. El diagnóstico se realiza en base al examen físico y la nasofaringoscopía. Al examen intraoral se observa falta de comunicación oronasal por presencia de cicatriz que fusiona borde libre del velo a la pared posterior de la faringe y ausencia de úvula (Figura 1). La nasofaringoscopía revela estenosis orofaríngea severa con pasaje filiforme asociado a bridas esofágicas. Éstas reemplazan los pilares amigdalinos y elevan la lengua hasta el nivel del paladar duro. 


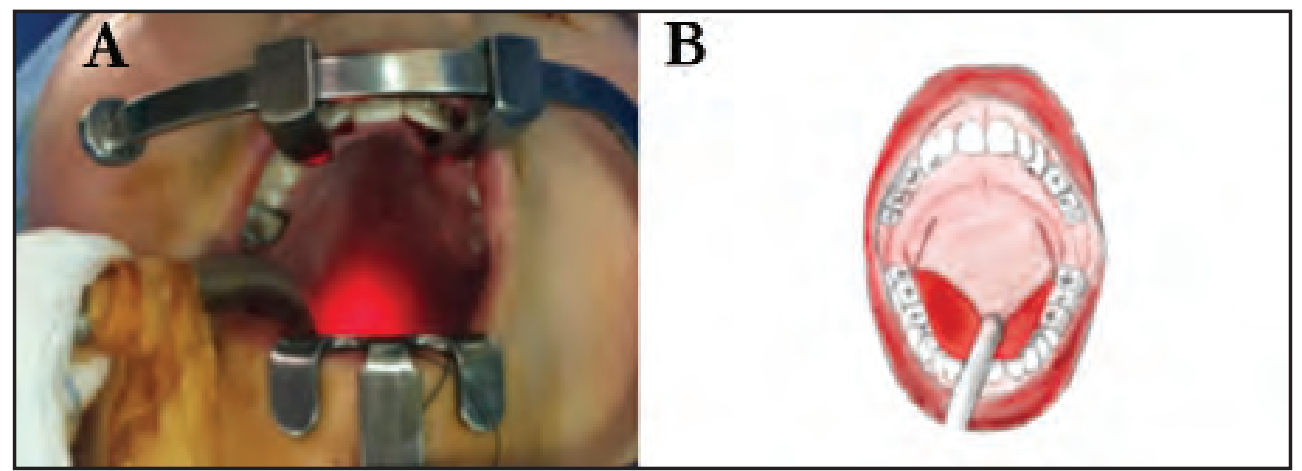

Figura 1. Caso clínico 1. A. Estenosis faríngea visión directa. B. Diagrama de estenosis faríngea.

\section{CASO CLÍNICO 2}

Paciente de sexo femenino. Se realiza adenoamigdalectomía a los 3 años de edad. Es derivada para su estudio y manejo por presentar síntomas de obstrucción nasal. La nasofibroscopía muestra adherencia del pilar posterior hacia la retrofaringe. Se observa una importante cicatriz fibrosa, compatible con estenosis faríngea posquirúrgica.

\section{CASO CLÍNICO 3}

Paciente de sexo masculino, con antecedente de adenoamigdalectomía a los 5 años de edad. Cursa con estenosis faríngea posquirúrgica. Inicialmente se realiza faringoplastía por su equipo tratante, la cual se reestenosa. Es derivado a nuestro centro, donde se constata fusión completa retrofaríngea no logrando traspasar la zona con el nasofibroscopio. Para los casos descritos se diseña un protocolo quirúrgico (Tabla 1), que consiste en visión directa de la zona estenosada por boca, complementando con la colocación del endoscopio con fuente de luz por nariz para confirmar la altura o longitud del defecto (zona adherida). Infiltración local con solución de bupivacaína 0,25\% y adrenalina en una dilución de 1:100.000. Se realiza incisión en zona fusionada y se liberan adherencias mediante zetaplastías y colgajos en VY para ampliar el espacio (Figura 2).

Se comprueba bajo visión endoscópica la permeabilidad del trayecto desde las coanas hasta la orofaringe. En todos los casos se diseña un colgajo de pared faríngea lateral, el cual se rota

Tabla 1. Protocolo de estudio y evaluación para casos de estenosis faríngea posamigdalectomía

\begin{tabular}{|l|l|}
\hline PROTOCOLO & $\begin{array}{l}\text { Evaluación clínica } \\
\text { Nasofaringoscopía }\end{array}$ \\
\hline Preoperatorio & Planificación intraoperatoria con visión mixta (oral y endoscópica nasal) \\
& Diseño de colgajo faríngeo de pared lateral interpuesto en zona estenótica \\
\hline Intraoperatorio & $\begin{array}{l}\text { Instalación de tutor a permanencia por } 15 \text { días } \\
\text { Evaluación con nasofaringoscopía más toma de impresión para bulbo } \\
\text { Posoperatorio }\end{array}$ \\
& Instalación de bulbo faríngeo por 6 meses \\
\hline
\end{tabular}


y se interpone en la zona cruenta generada en la pared posterior de la faringe cuidando de no dejar suturas en contacto de manera de evitar la reestenosis (Figura 3).

Se calibra la permeabilidad utilizando una sonda Nelaton \#12 French. El caso del paciente con la fusión retrofaríngea completa, dada la extensa fibrosis se planificó cirugía en 2 tiempos. En el primer tiempo quirúrgico se liberó el velo del paladar a derecha y 6 meses después se intervino para liberar a izquierda. Los pacientes se mantienen en el posoperatorio con tubo nasofaríngeo en forma de tutor por 15 días y posteriormente se les instala un bulbo faríngeo (Figura 4) que actúa como con-

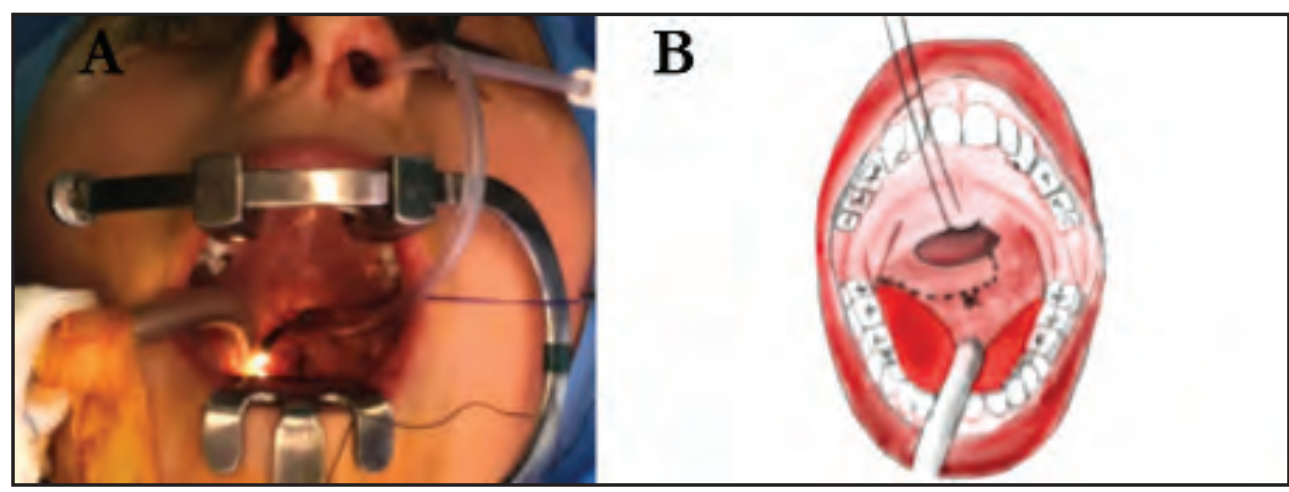

Figura 2. Caso clínico 1. A. Liberación de adherencias. B. Diagrama con liberación de adherencia y diseño de colgajo faríngeo lateral.

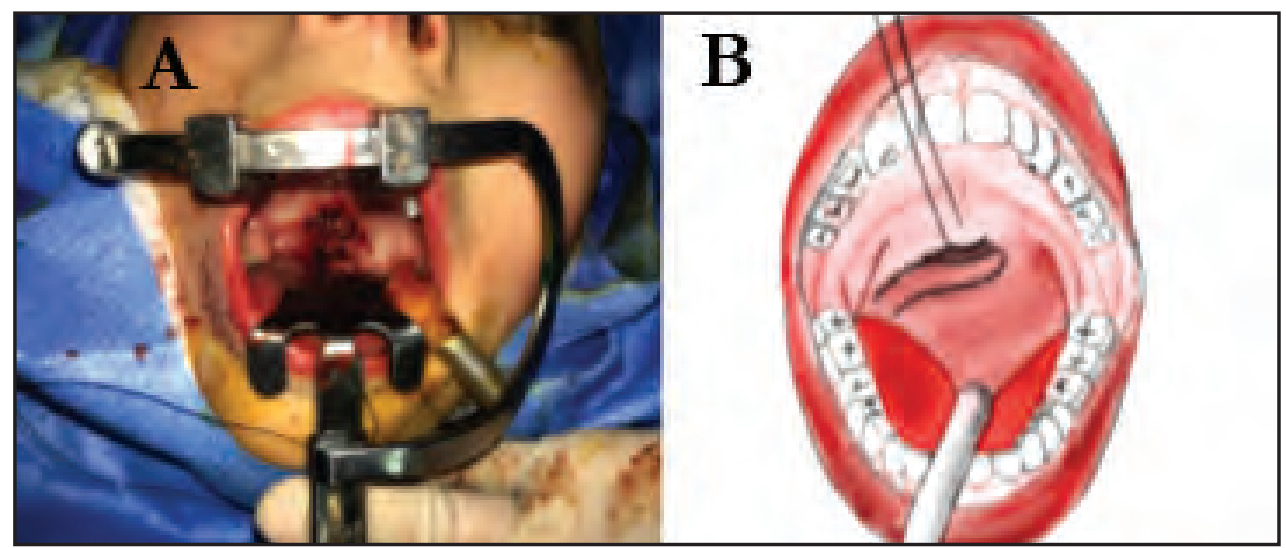

Figura 3. Caso clínico 1. A. Colgajo faríngeo interpuesto en zona cruenta. B. Diagrama de colgajo faríngeo interpuesto.

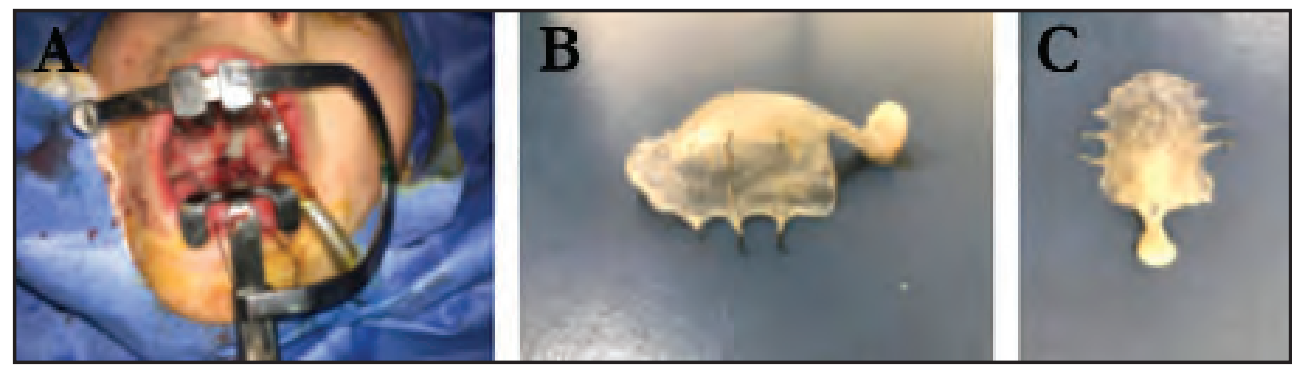

Figura 4. Caso clínico 1. A. Instalación de bulbo faríngeo posquirúrgico inmediato. B. Bulbo faríngeo visión lateral. C. Bulbo faríngeo visión superior. 
formador. El seguimiento a largo plazo se realiza mediante examen físico y nasofibroscopía de control evidenciando la mantención de permeabilidad oronasal (Tabla 2).

\section{DISCUSIÓN}

La estenosis faríngea es una complicación infrecuente posadenoamigdalectomía y se define como el estrechamiento de la comunicación normal entre la nasofaringe y la orofaringe. Ésta puede presentar distintas manifestaciones clínicas que dependen del grado de compromiso de las estructuras anatómicas afectadas generando un problema obstructivo mecánico y funcional. Clásicamente se describe a la región nasofaríngea como una estructura cuboides, cuyo techo corresponde a la curvatura craneal, que cae en forma inclinada hacia atrás continuando con las estructuras que cubren las dos primeras vértebras cervicales. El límite anterior está constituido por las coanas, mientras que el piso por el velo palatino. Los movimientos básicos del velo palatino son producidos por el músculo tensor y el músculo elevador del velo, cuyo objetivo es el contacto con la pared posterior de la faringe al contraerse su musculatura. Estos movimientos conducen a un correcto acoplamiento de la cavidad nasal y oral para generar una adecuada emisión de la voz. Así mismo, el movimiento de la lengua y los músculos constrictores de la faringe propician el mecanismo de deglución. En la estenosis faríngea, la adhesión cicatricial del velo palatino, pilares amigdalinos y/o base de la lengua alteran la funcionalidad, produciendo trastornos de la función nasal, drenaje tubárico, roncopatía, trastornos del sueño, trastornos de deglución y alteración de la resonancia por disfunción velofaríngea.

Los pacientes que presentan esta complicación generalmente debutan en forma insidiosa dentro de las primeras semanas posquirúrgicas con síntomas como obstrucción nasal, respiración oral, roncopatía, cambios en la resonancia (hiponasalidad) y disfagia. Clínicamente se aprecia como un tejido cicatricial que inmoviliza el velo, bridas que fijan los pilares amigdalinos y adherencias en relación a la lengua asociados a una apertura diminuta. La técnica diagnóstica de elección para estos casos corresponde a la nasofibroscopía flexible que además informa la extensión de la complicación y orienta a la elección de la alternativa de tratamiento quirúrgico.

Se han descrito varias causas de estenosis faríngea, en relación a infecciones, cáusticos y complicaciones posquirúrgicas. Los factores de riesgo asociados a la formación de adherencias posadenoamigdalectomía incluyen, sangrado importante, zonas cruentas extensas, predisposición a formar queloides, disección profunda de la región inferior de las amígdalas con escisión de amígdalas linguales, grandes bandas de adenoides, cirugías en multiniveles, uso excesivo de electrocoagulación y reoperaciones ${ }^{4,7-9}$. Hay múltiples alternativas terapéuticas, que van desde tratamientos menos invasivos como inyección local de corticoides, remoción de adherencias, y uso de obturador farín-

Tabla 2. Resumen de hallazgos y procedimiento quirúrgico de los 3 casos clínicos

\begin{tabular}{|llll|}
\hline & Caso 1 & Caso 2 & Caso 3 \\
\hline Edad & 3 años & 3 años & 5 años \\
\hline Hallazgos prequirúrgicos & $\begin{array}{l}\text { Cicatriz que fusiona borde libre } \\
\text { del velo a la pared posterior } \\
\text { de la faringe. }\end{array}$ & $\begin{array}{l}\text { Cicatriz fibrosa con } \\
\text { adherencia pilar } \\
\text { posterior a la faringe }\end{array}$ & $\begin{array}{l}\text { Fusión completa } \\
\text { retrofaríngea }\end{array}$ \\
\hline Procedimiento quirúrgico & $\begin{array}{l}\text { Liberación bridas } \\
+ \text { colgajo de rotación. } \\
\text { (1 tiempo quirúrgico) }\end{array}$ & $\begin{array}{l}\text { Liberación bridas } \\
+ \text { colgajo de rotación. } \\
(1 \text { tiempo quirúrgico) }\end{array}$ & $\begin{array}{l}\text { Liberación bridas } \\
+ \text { colgajo de rotación } \\
\text { a derecha. } \\
\text { (2 tiempos quirúrgicos) }\end{array}$ \\
\hline Permeabilización retrofaríngea & Sí & Sí & Sí \\
\hline
\end{tabular}


geo; hasta procedimientos más extensos incluyen el uso de colgajos locales, regionales o incluso colgajos libres ${ }^{4-6,10}$. Para los casos descritos se realizó liberación de adherencias y plastía de las zonas con el objetivo de ampliar el espacio, asociado a un colgajo de pared faríngea lateral, el cual se interpone en la zona cruenta de manera de evitar reestenosis.

\section{CONCLUSIÓN}

Cada caso debe ser individualizado debido a la importante variación que hay en la longitud y

\section{BIBLIOGRAFÍA}

1. David JP, Claudia HC. Complications of tonsillectomy: Evidence based review. Revista de otorrinolaringología y cirugía de cabeza y cuello 2015; 75: 275-85.

2. Andrés aV, Marcel SC, Cecilia SM, Romina GV. Amigdalectomía y adenoidectomía: Conceptos, técnicas y recomendaciones. Revista de otorrinolaringología y cirugía de cabeza y cuello 2016; 76: 99-110.

3. McDonald TJ, Devine KD, Hayles AB. Nasopharyngeal Stenosis Following Tonsillectomy and Adenoidectomy: Report of Six Cases and Their Repair. Archives of Otolaryngology 1973; 98: 38-41.

4. Sмiтh ME. Prevention and treatment of nasopharyngeal stenosis. Operative Techniques in Otolaryngology-Head and Neck Surgery 2005; 16: 242-7.

5. Santos V, Ruffy M, Polisar I. Stenosis of the oropharynx treated with intralesional triamcinolone. Ear, nose, \& throat journal 1977; 56: 164-7. calibre de la zona estenosada. La técnica de colgajo faríngeo lateral tiene por objetivo interponer mucosa faríngea sana para cobertura de lecho cruento y constituye una alternativa terapéutica eficaz para resolver la estenosis faríngea posquirúrgica. Posteriormente se mantiene un bulbo faríngeo por aproximadamente 6 meses, que se va modificando con un acondicionador de tejidos para lograr calibre apropiado de la vía aérea y evitar recidivas. En todos los pacientes se realiza un seguimiento a largo plazo, control con nasofibroscopía y evaluación de la voz por fonoaudiología.

6. Mann RJ, O’Brien AL, Adams NS, Girotto Ja, Polley JW. Repair of Oropharyngeal Stenosis with Bilateral Buccal Myomucosal Flaps. Annals of Plastic Surgery 2017; 79: 162.

7. Giannoni C, Sulek M, Friedman EM, Duncan NO. Acquired Nasopharyngeal Stenosis: A Warning and Review. Archives of Otolaryngology-Head \& Neck Surgery 1998; 124: 163-7.

8. Woolf RM, Broadbent RT. Nasopharyngeal stenosis following tonsillectomy and adenoidectomy. Plastic and Reconstructive Surgery 1970; 45: 352-5.

9. Prager JD, Hopkins BS, Propst EJ, Shott SR, Cotton RT. Oropharyngeal Stenosis: A Complication of Multilevel, Single-Stage Upper Airway Surgery in Children. Archives of Otolaryngology-Head \& Neck Surgery 2010; 136: 1111-5.

10. Wan DC, Kumar A, Head CS, Katchikian H, Bradley JP. Amelioration of Acquired Nasopharyngeal Stenosis, with Bilateral Z-Pharyngoplasty. Annals of Plastic Surgery 2010; 64: 747-50. 